\section{Service standards for sexual health} Madam

We fail to understand why Dr Stephen Searle ${ }^{1}$ feels that implementing the Faculty's Service Standards should detract from our level of client care. If service has a clear chaperone policy or protocol then the amount of actual documentation required is minimal. An entry in the case notes "chaperone declined' or 'chaperone: Nurse Smith' should suffice to indicate adherence with the policy.

In Abacus Clinics in Liverpool we established a chaperone policy in 2001 in response to guidance from the General Medical Council ${ }^{3}$ and the Royal College of Obstetricians and Gynaecologists. ${ }^{4}$ This followed a lengthy in-house discussion an required a significant 'culture change' for a predominantly female staff who previously viewed the offer of a chaperone as a purely gender issue with medico-legal implications. Some felt that the offer of a chaperone would alarm clients and make them suspicious of the clinician. There were concerns about the chaos that would ensue in busy clinics if all clients wanted a chaperone. In the event, these fears were unfounded. A review of staff perspectives on the policy a year after its introduction showed that the majority of staff felt that less than $5 \%$ of clients accepted a chaperone when offered. It was felt that the reason for requesting a chaperone had more to do with relieving the client's anxiety about the examination rather than concerns about unprofessional behaviour by the clinician. Whilst only $18 \%$ of staff members stated that they always offered a chaperone, up to $80 \%$ usually or sometimes did so. The main reason given for not offering a chaperone was that they simply forgot to do so because it was a change to their previous routine practice. Those who did offer documented the offer on mos occasions. There was no evidence to suggest that implementing the policy had a significan detrimental effect on clinic times or workload.

Documentation relating to practical procedures, e.g. fitting an intrauterine device (IUD), may be more time consuming but it is important, not just for medico-legal reasons, but for ensuring continuity of good clinical care and risk management. Perhaps the devil is in the detail. It is up to us as clinicians to decide what is and what is not essential documentation Following an audit ${ }^{5}$ of relevant case notes within our service, carried out in 2000, we established a minimum standard ${ }^{6}$ for documentation relating to IUD insertion acceptable to all our clinicians. In ou experience staff have been happy to implement these standards, accepting them as a useful aid to maintaining good clinical care.

Standards achieved by consensus should serve to protect both client and clinician.

Laraine Murray, RGN, Dip. Health Education Nursing

Family Planning Nurse, Abacus Clinics for Contraception and Reproductive Health Care Liverpool, UK

James T McVicker, MRCGP, MFFP

Associate Specialist, Abacus Clinics for Contraception and Reproductive Health Care, North Liverpool PCT, 40-46 Dale Street, Liverpool L2 $5 S F, U K$ Searle S. Service standards for sexual health (Letter).
I Fam Plann Reprod Health Care 2004; 30(2): 131 . Faculty of Family Planning and Reproductive Health Care (FFPRHC). Service Standards for Sexual Health Services. hent///Www.Ifprhc.org.uk. Examinations. London, UK: GMC, December 2001. http://www omc-uk.

Royal College of Obstetricians and Gynaecologists (RCOG). Intimate Examinations. Report of a Working Party. London, UK: RCOG, September 1997. http://www.rcog.org.uk.

Murray L. A review of staff attitudes to the implementation of a chaperone policy in a large community based contraceptio and reproductive health care service. Unpublished audit.

6 Kasliwal AP, Webb AMC. Intrauterine device insertion: setting our standards. J Fam Plann Reprod Health Care 2002;
Madam

I would like to respond to the letter by Stephen Searle in the April 2004 issue of the Journal on behalf of the Clinical Standards Committee of the Faculty. ${ }^{1}$ The reason d'etre of the National Health Service, and for all who work in it, is to provide high-quality, continuously improving, patientcentred care. In a relatively short space of time clinical governance has become a pre-eminent tool in enabling this to happen.

Whilst the number of publications on this subject is almost overwhelming, the basic principles applied to clinical practice should ensure the delivery of good care. The service standards are produced by the Faculty with the object of interpreting national guidance and directives and incorporating these with core clinical governance principles to provide specialty-specific standards. They are intended to aid clinicians in patient care. Clear record keeping is fundamental to, and an integral part of, patien care. To view it as reactive bureaucracy, which is only necessary to protect in cases of legal action for poor practice, is surely to miss the point. Rather, good record keeping is a fundamental part of each episode of patient care.

Clearly formation of standards is a rapidly developing area. The documents produced by the Clinical Standards Committee have short review cycles so that views can be included commensurate with this progression. Further, the Committee always welcomes comments and suggestions. It is to be hoped that these will inform the refinement of the standards at review thus maximising their usefulness.

Christine Robinson, FRCOG, MFFP

Chair of the Clinical Standards Committee, Faculty of Family Planning and Reproductive Health Care, London, UK

Reference
$1 \quad$ Searle S. Service standards for sexual health (Letter).

J Fam Plann Reprod Health Care 2004; 30(2): 131.

\section{IUD insertion following medical TOP}

Madam

We found the FFPRHC Guidance on 'The copper intrauterine device as long-term contraception' most informative but were surprised by the lack of data relating to intrauterine device (IUD) insertion following medical termination of pregnancy (TOP) (Section 26). Our district general hospital performs more than 300 medical TOPs annually up to 83 days' gestation. All women are screened for sexually transmitted infections and there is a $96 \%$ complete miscarriage rate. ${ }^{2}$ In a proportion of cases abortion occurs or completes at home in the first few days following the administration of misoprostol. If abortion occurs in hospital, contraception such as oral contraceptives or DepoProvera ${ }^{\circledR}$ is commenced immediately by the nursing staff. Women are then reviewed in a weekly specialist family planning clinic approximately 7-10 days after their termination procedure. This review ensures that the termination is complete and allows the patient's physical and emotional status to be assessed. IUDs or implants are inserted at this visit. Occasionally at this review appointment bleeding is still continuing and further misoprostol is required to expel all products of conception. Another appointment is then made 1 week later for the IUD fitting.

Since January 2000, 55 copper IUDs have been inserted between 4 and 30 (average, 11) days following medical TOP. The majority were Gyne T380 ${ }^{\circledR}$ or Nova T380 ${ }^{\circledR}$ IUDs but included two GyneFix ${ }^{\circledR}$ IUDs in 2000 and two Flexi-T300 ${ }^{\circledR}$ IUDs in 2003. The two women whose copper IUDs were fitted at 29 and 30 days post-TOP had had continued problems with bleeding and required further doses of misoprostol. Thirty Mirena ${ }^{\circledR}$ intrauterine systems (IUS) were also inserted 6-16 (average, 10) days following medical TOP. There have been no difficulties or immediate complications with insertions using this policy.

In 2001, a Mirena IUS was partially expelled
20 days after insertion and a new IUS was refitted without incident. Two women have conceived with copper IUDs in situ 4 and 6 months after nsertions. One subsequently miscarried and the second has had a further TOP. A third woman had an ectopic pregnancy 14 months after IUD fitting.

We consider that the Guidance of the Clinical Effectiveness Unit (CEU) that IUDs should be fitted within 48 hours or after 4 weeks for women undergoing first-trimester medical TOP is too restrictive. The first-trimester postabortal uterus does not appear to behave like a postpartum uterus. In practice many women would not wish to be examined within the first 48 hours when the bleeding may be heavier and in some women the uterus may not be completely empty. Waiting for 4 weeks (presumably until after the next menses) requires women to arrange a further appointment that they may have difficulty keeping and also denies them efficient contraception for the first month after TOP.

We suggest that a review appointment, usually at 7-10 days post-medical TOP, allows safe insertion of both copper IUDs and Mirena IUS and should be promoted.

N Selvakumari, DFFP

Staff Grade, Maternity \& Women's Health Care, Royal Bolton Hospital, Bolton, UK

\section{E Stevenson, MFFP}

Clinical Assistant, Maternity \& Women's Health Care, Royal Bolton Hospital, Bolton, UK

M Cooper, DFFP

Clinical Assistant, Maternity \& Women's Health Care, Royal Bolton Hospital, Bolton, UK

M Tasker, MFFP

Consultant Gynaecologist, Maternity \& Women's Health Care, Royal Bolton Hospital, Minerva Road, Farnworth, Bolton BLA OJR, UK

References

FFPRHC Clinical Effectiveness Unit. FFPRHC Guidance (January 2004). The copper intrauterine device as long-term contraception. J Fam Plann Reprod Health Care 2004; Basu R, Gun

Bas R, Gunlach T, Tasker M. Mifepristone and misoprostol flexible regimen. J Fam Plann Reprod Health Care 2003; 29(3): 139-141. J Fam Plann Reprod Health Care 2003;

\section{Reply}

Madam FFPRHC Clinical Effectiveness Unit (CEU) provides evidence-based Guidance documents on contraceptive and reproductive health topics. The recent Guidance document 'The intrauterine device as long-term contraception' ${ }^{1}$ was developed using best available evidence from a systematic literature review, the collective knowledge of the multidisciplinary expert group and subsequent peer review. Despite a large number of medical abortions performed each year in England, Wales and Scotland, there is a lack of published evidence on the timing of intrauterine contraceptive insertion following medical abortion.

The insertion of intrauterine contraception immediately following abortion clearly has advantages. The insertion of intrauterine contraception at the time of surgical abortion is practical and safe. ${ }^{2}$ The World Health Organization (WHO) Medical Eligibility Criteria for Contraceptive Use (WHOMEC) ${ }^{3}$ recommends that intrauterine contraception can be inserted immediately following induced or spontaneous first-trimester abortion (WHO 1: unrestricted use). Although the risk of expulsion of an intrauterine device (IUD) following second-trimester abortion is increased, ${ }^{2}$ WHOMEC recommends that the benefits still outweigh the risks (WHO 2) 3 Although WHOMEC does not provide recommendations regarding insertion of intrauterine contraception in the weeks following abortion, evidence from case-control studies showed low perforation rates with insertion within 30 days of abortion. ${ }^{4}$ 